\title{
The economic burden of medical care in general and sleep apnea syndrome in particular
}

\author{
Vidya Krishnan
}

Received: 21 April 2009 /Accepted: 25 April 2009/Published online: 30 May 2009

(C) Springer-Verlag 2009

Healthcare spending in the U.S. has been growing steadily for the last 30 years, at a rate $2 \%$ faster than the rest of the economy. The rising costs of healthcare have had its toll on individuals' and the nation's finances. Nearly a half-million people file for bankruptcy every year because of high medical costs. In 2007, the total U.S. healthcare bill came to $\$ 2.3$ trillion-more than was spent on food in the same year. Despite this relatively high level of spending, the U.S. does not appear to provide substantially greater health resources to its citizens, or achieve substantially better health benchmarks, compared to other developed countries [1]. As has been clear for the last 10 years, it is high time to reform our healthcare delivery system, to make it more effective and efficient.

Systematic economic analyses of healthcare delivery are and will be a valuable tool in reshaping our healthcare system. Alongside research that provides the evidence to base medical decision-making should be the research that determines the cost impact of the medical decisions. Many of our current healthcare policies and practices in medicine are guided by consensus opinion, instead of informed economic analyses. Such analyses require data on the scope, the consequences, and the costs of a medical disorder. Obstructive sleep apnea (OSA) is a disorder that has been increasingly identified as one that is highly prevalent, has significant impact on quality of life, is a risk factor for multiple co-morbid conditions and increased mortality, and is relatively simple to treat. Our imperative in

\footnotetext{
V. Krishnan $(\square)$

Division of Pulmonary, Critical Care and Sleep Medicine,

MetroHealth Medical Center,

Room BG3-37, 2500 MetroHealth Drive,

Cleveland, OH 44109, USA

e-mail: vkrishnan@metrohealth.org
}

the Sleep Medicine community is to convince policy makers that allocating resources to the diagnosis and treatment of OSA will have a substantial positive impact, both on medical outcomes and overall medical costs [2].

Treatment of sleep apnea syndrome (SAS), defined as OSA with clinical sleepiness, is both economical and effective. A number of studies have supported the economic benefits of treating SAS, particularly when comparing the incremental costs of treatment with CPAP per quality adjusted life years. When quality of life, costs of therapy, and motor vehicle outcomes are considered, CPAP therapy for patients with SAS is attractive for both economics and the health benefits $[3,4]$. These studies do not address the potential benefits of future medical cost savings due to improved health status and reduction of co-morbidities. The economical benefits of CPAP may prove to be even more advantageous in subsets of patients who may be more vulnerable to the complications of SAS.

While current medical literature justifies the treatment of all patients with SAS, limited healthcare resources may be best allocated to the subsets of patients that may benefit most from the diagnosis and treatment. Patients with obstructive lung disease, such as chronic obstructive pulmonary disease (COPD) and asthma, may be disproportionately affected by the presence of SAS. Patients with concomitant COPD and SAS have been shown to have more frequent nocturnal desaturations and sleep fragmentation [5]. In patients with severe asthma and OSA, treatment of the OSA with CPAP has been shown to significantly improve nocturnal asthma symptoms, also suggesting an interaction between the two disorders $[6,7]$. There is sufficient reason to believe that the presence of SAS in patients with obstructive lung disease may worsen health status and increase the healthcare needs of these patients. 
In this issue, Shaya and colleagues present data from a retrospective analysis of Medicaid beneficiaries with obstructive lung disease [8]. The authors' aim was to determine the incremental economic burden of SAS in patients with COPD and/or asthma. They found that SAS was more commonly listed as a co-morbid condition in patients with asthma and COPD, as compared to either condition alone. This may be a reflection of more severe respiratory limitation, or possibly that patients who were seen in the healthcare system more often were more likely to be identified with having a sleep disorder. Additionally, patients with COPD (with or without asthma) had significantly higher medical utilization (as measured by medical service claims) and estimated costs when SAS was also present. Patients with asthma alone, however, were not found to have this differential in medical utilization or costs based on the presence of SAS. These data suggest that patients with COPD may be an important subset of patients who are more profoundly affected by the negative impact of SAS and who may benefit the most (both economically and in terms of health status) from treatment of SAS.

Why are COPD patients, but not asthma patients, with SAS more likely to have increased medical service requirements and medical costs? One reason may be that their health status may be more fragile than patients with asthma. Patients with COPD are more likely to be older (as is true in the current cohort of patients), and therefore have more co-morbidities, than patients with asthma alone. Additional co-existing disease processes, such as SAS, has more of a potential to worsen the patient's overall health. In a similar patient population as in the present study, patients with COPD (with or without asthma) were more likely to require inpatient services and had a higher medical utilization overall [9]. Another potential reason is that asthma patients may have been less likely to be screened for SAS than patients with COPD. The additional costs and medical utilization required to diagnose and treat SAS may have been provided for the patients who were more likely to have risk factors for SAS. The patients with COPD were more likely to be older and male, which are identified risk factors for SAS. While this may explain how the direct costs of management of SAS contribute to the elevated resource utilization and costs for COPD patients, another explanation might be a difference in disease interaction of SAS on the obstructive lung diseases. The fact that presence of SAS increases medical utilization and costs in COPD patients suggests that SAS may worsen the overall health status of patients with COPD. Conversely, in patients with asthma, the co-morbid presence of SAS does not seem to influence health costs or utilization, suggesting asthma symptomatology and severity may be independent of SAS. While these considerations are intriguing, the findings of this retrospective study need to be confirmed with prospective analyses of a broader patient population.

Cost analyses of healthcare are the cornerstone for developing effective healthcare policy. The finding that COPD patients with SAS utilize more healthcare resources identifies a patient population for whom targeted therapy of disease may reduce healthcare costs in total. Until the ultimate goal of treating all patients with SAS can be achieved, comprehensive economic analyses will guide our allocation of resources to realize the greatest benefit of treatment of SAS for individuals and our community.

\section{References}

1. Anderson GF, Frogner BK, Johns RA, Reinhardt UE (2006) Health care spending and use of information technology in OECD countries. Health Affairs 25(No. 3):819-831

2. Kapur VK, Alfonso-Cristancho R (2009) Just a good deal or truly a steal? Medical cost savings and the impact on the cost-effectiveness of treating sleep apnea. Sleep 21:135-6

3. McDaid C, Griffin S, Weatherly H et al (2009) Continuous positive airway pressure devices for the treatment of obstructive sleep apnoea-hypopnoea syndrome: a systematic review and economic analysis. Health Technol Assess 13(4):1-119

4. Weatherly HL, Griffin SC, McDaid C et al (2009) An economic analysis of continuous positive airway pressure for the treatment of obstructive sleep apnea-hypopnea syndrome. Int J Technol Assess Health Care 25(1):26-34

5. Sanders MH, Newman AB, Haggerty CL, Redline S, Lebowitz M, Samet J, O'Connor GT, Punjabi NM, Shahar E, for the Sleep Heart Health Study (2003) Sleep and sleep-disordered breathing in adults with predominantly mild obstructive airway disease. Am J Respir Crit Care Med 167:7-14

6. Chan CS, Woodcock AJ, Sullivan CE (1988) Nocturnal asthma: role of snoring and obstructive sleep apnea. Am Rev Respir Dis 137:1502-1504

7. Ciftci TU, Ciftci B, Guven SF et al (2005) Effect of nasal continuous positive airway pressure in uncontrolled nocturnal asthmatic patients with obstructive sleep apnea syndrome. Respir Med 99:529-534

8. Shaya FT, Lin P-J, Aljawadi MH, Scharf SM (2009) Elevated economic burden in obstructive lung disease patients with concomitant sleep apnea syndrome. Sleep Breath. doi:10.1007/ s11325-009-0266-2

9. Shaya FT, Dongyi D, Okazawa MO et al (2008 Jul) Burden of concomitant asthma and COPD in a Medicaid population. Chest 134(1):14-9 